\title{
Mid-term study of transcatheter aortic valve implantation in an Asian population with severe aortic stenosis: two-year Valve Academic Research Consortium-2 outcomes
}

\author{
Nicholas Chew ${ }^{1}$, PhD, Jimmy Kim Fatt Hon ${ }^{1}$, MBChB, Wei Luen James Yip ${ }^{1}$, MBBS, Siew Pang Chan ${ }^{1}$, PhD, \\ Kian-Keong $\underline{P o h}^{1}$, MBBChir, William Kok-Fai Kong ${ }^{1}$, MBChB, Kristine Leok Kheng Teoh ${ }^{1}$, MBBChir, Tiong Cheng $\underline{\text { Yeo }}^{1}$, MBBs, \\ Huay Cheem $\underline{T a n}^{1}$, MBBS, Edgar Lik Wui Tay ${ }^{1}$, MBBS
}

INTRODUCTION Transcatheter aortic valve implantation (TAVI) is an effective treatment for high-risk or inoperative patients with severe aortic stenosis. Given the unique characteristics of Asian populations, questions regarding mid-term outcomes in Asians undergoing TAVI have yet to be addressed. We evaluated the two-year clinical outcomes of TAVI in an Asian population using Valve Academic Research Consortium-2 definitions.

METHODS This prospective study recruited 59 patients from a major academic medical centre in Singapore. The main outcomes were two-year survival rates, peri-procedural complications, symptom improvement, valvular function and assessment of learning curve.

RESULTS Mean age was 76.8 years ( $61.0 \%$ male), mean body surface area $1.6 \mathrm{~m}^{2}$ and mean logistic EuroSCORE $18.7 \%$. Survival was $93.2 \%, 86.0 \%$ and $79.1 \%$ at 30 days, one year and two years, respectively. At 30 days post TAVI, the rate of stroke was $1.7 \%$, life-threatening bleeding $5.1 \%$, acute kidney injury $25.0 \%$, major vascular complication $5.1 \%$, and new permanent pacemaker implantation $6.8 \% .29 .3 \%$ of TAVI patients were rehospitalised (47.1\% cardiovascular-related) within one year. These composite outcomes were measured: device success (93.2\%); early safety (79.7\%); clinical efficacy (66.1\%); and time-related valve safety (84.7\%). Univariate analysis found these predictors of two-year all-cause mortality: logistic EuroSCORE (hazard ratio [HR] 1.07; $<$ < 0.001); baseline estimated glomerular filtration rate $(H R$ 0.97; $p=0.048)$; and acute kidney injury (HR 5.33; $p=0.022$ ). Multivariate analysis identified non-transfemoral TAVI as a predictor of cardiovascular-related two-year mortality (HR 14.64; $p=0.008$ ).

CONCLUSION Despite the unique clinical differences in Asian populations, this registry demonstrated favourable midterm clinical and safety outcomes in Asians undergoing TAVI.

Keywords: aortic stenosis, transcatheter aortic valve implantation

\section{INTRODUCTION}

Despite the poor prognosis associated with severe aortic stenosis (AS), it has been found that a significant number of Asian patients would still refuse conventional open-heart surgery. ${ }^{(1)}$ The reasons for refusing surgery despite favourable outcomes remain undetermined. We speculate that the less-invasive transcatheter aortic valve implantation (TAVI) may be rapidly adopted in this population.

The landmark PARTNER trial found that TAVI was effective in reducing mortality and rehospitalisation, and improved symptoms, with preservation of valvular function, for up to five years. ${ }^{(2)}$ However, questions regarding the clinical outcomes of TAVI in an Asian population have yet to be adequately addressed. Demographic studies in Singapore have revealed unique characteristics of patients suffering from severe AS that may be applicable to the wider Asian population. The size of Asian patients' aortic annulus tends to be smaller than that reported in other parts of the world. This would have implications for the selection of device sizes appropriate for this population, as well as the clinical outcomes of the TAVI procedure. ${ }^{(1)}$ This study represents the first systematic prospective study in Asia to evaluate the mid-term outcomes of TAVI in an Asian population. It utilises the Valve Academic Research Consortium-2 (VARC-2) consensus definition for outcomes, ${ }^{(3)}$ which allows for adequate comparison against other published studies.

\section{METHODS}

Between 2010 and 2015, patients who presented at a major academic medical centre in Singapore with severe symptomatic AS and were deemed by the heart team to be with high surgical risk or inoperable but eligible for TAVI were recruited consecutively into this prospective registry. The logistic EuroSCORE (European System for Cardiac Operative Risk Evaluation) and the Society of Thoracic Surgeons' Predicted Risk of Mortality score were recorded and used as general risk assessment tools. The final consensus to undergo the TAVI procedure was made by the heart team, comprising two experienced interventional cardiologists,

${ }^{1}$ National University Heart Centre, Singapore

Correspondence: Asst Prof Edgar Tay, Senior Consultant, National University Heart Centre, 1E Kent Ridge Road, NUHS Tower Block, Level 9 , Singapore 119228. edgar_tay@nuhs.edu.sg 
two cardiac surgeons, imaging cardiologists and radiologists. The diagnosis of AS was made according to American College of Cardiology guidelines. ${ }^{(4)}$

The clinical and demographic characteristics of patients were obtained from electronic medical records. Baseline characteristics were presented as frequencies and percentages for categorical variables and mean \pm standard deviation for continuous variables. Important baseline measurements were extracted from the hospital's echocardiographic and radiological web database. Echocardiographic measurements of the severity of AS and valve function were performed as recommended by the VARC-2 guidelines. Measurements of the computed tomography (CT) annular dimension were performed as per previously published techniques. ${ }^{(5)}$ This study received ethics approval from the institutional review board. All patients gave their written consent for the study.

The valves used were as follows: 49 SAPIEN XT (Edwards Lifesciences, Irvine, CA, USA), two CoreValve (Medtronic Inc, Minneapolis, MN, USA), two CoreValve Evolut ${ }^{\mathrm{TM}} \mathrm{R}$ (Medtronic Inc), one Lotus ${ }^{\mathrm{TM}}$ Valve System (Boston Scientific, Natick, MA, USA), two Engager ${ }^{\mathrm{TM}}$ aortic valve bioprostheses (Medtronic Inc), one SAPIEN 3 valve (Edwards Lifesciences Inc) and two JenaValve (JenaValve Technologies $\mathrm{GmbH}$, Munich, Germany). The same heart team reviewed all 59 patients and came to a consensus regarding the appropriate valve type and size, with the help of CT-guided annular sizing, for each individual patient. $\mathrm{CT}$ assessments were done by two dedicated CT radiologists. Transfemoral (TF), transapical and transaortic techniques were performed as previously described..$^{(6-8)}$ We adopted a 'TF first' approach and only used the transapical route if the TF technique was found to be unsuitable for the patient. There was only one case of transaortic TAVI. The first eight cases were done in the cardiac catheterisation laboratory, and subsequent cases were done in a hybrid operating room. Follow-up assessments of the prosthetic valve and left ventricular parameters were obtained from serial echocardiography.

All study outcomes were defined according to the VARC-2 recommendations. ${ }^{(3)}$ The primary outcome of the study was the two-year survival rate of patients who underwent TAVI. Secondary outcomes included all-cause mortality, myocardial infarction, stroke, bleeding complications, acute kidney injury $(\mathrm{AKI})$, conduction disturbances and arrhythmias, other TAVIrelated complications, and prosthetic valve dysfunction. The latter included severe prosthesis-patient mismatch (PPM), which was defined as transcatheter valve effective orifice area $<0.65 \mathrm{~cm}^{2} / \mathrm{m}^{2}$, as well as paravalvular leak (PVL). These secondary outcomes were analysed within a 30-day period. Rehospitalisation rates and symptom status, determined by New York Heart Association (NYHA) class, were recorded at one year of follow-up.

Composite outcomes, as described by the VARC-2 criteria, were also assessed. Device success was defined as the absence of procedural death, the correct placement of the prosthetic heart valve into the correct anatomical site, the performance of the prosthetic heart valve as measured by the PPM, mean aortic valve gradient $<20 \mathrm{mmHg}$ or peak velocity $<3 \mathrm{~m} / \mathrm{s}$, and the absence of moderate or severe PVL. Early safety at 30 days was defined as all-cause mortality, all strokes or life-threatening bleeding, Stage 2 or $3 \mathrm{AKI}$, major vascular complications, coronary artery obstruction or valve dysfunction requiring repeated procedure. Clinical efficacy after 30 days was based on all-cause mortality, all strokes, rehospitalisation for valve-related problems, exacerbated congestive heart failure or valve-related dysfunction (aortic mean valve gradient $\geq 20 \mathrm{mmHg}$, aortic valve area (AVA) $\leq 0.9-1.1 \mathrm{~cm}^{2}$ and/or moderate or severe PVL). Time-related valve safety was determined by structural valve deterioration, thromboembolic events, prosthetic valve endocarditis or thrombosis. ${ }^{(3)}$

The recruitment period for this study was from November 2010 to July 2015. Prospective follow-up was done during clinic visits, and follow-up ended on 13 August 2015. This gave a minimum follow-up time of one month and a maximum of five years. Due to the variability of the follow-up period among the patients, only 59, 57 and 43 patients were followed up at 30 days, one year and two years, respectively. All participants remained in contact until their death or the end of the study period.

Box and whisker plots were applied to compare patients' mean pressure gradient (MPG, in $\mathrm{mmHg}$ ) and AVA (in $\mathrm{cm}^{2}$ ) at baseline, 30 days and two years. Kaplan-Meier curves were constructed to examine time to mortality for all patients. The log rank test was used to ascertain whether there was a significant difference between those with logistic EuroSCORE $\leq 20 \%$ and $>20 \%$. Next, Cox proportional hazards regression analyses were performed to ascertain how the identified predictors could explain cardiovascular-related and all-cause mortality at two years. A graphical approach and global test were used to assess the validity of the proportional hazards assumption. All statistical tests were conducted at a $10 \%$ level of significance, considering the relatively small sample size, and analysed with Stata/MP version 14 (StataCorp, College Station, TX, USA) and SAS version 9.3.

\section{RESULTS}

A total of 59 patients were recruited for this study. Their mean age was $76.8 \pm 8.7$ years, mean body surface area (BSA) was $1.6 \pm 0.2 \mathrm{~m}^{2}$, and $36(61.0 \%)$ patients were male. The patients selected for the study had a high burden of coronary artery disease, peripheral arterial disease, chronic kidney disease, diabetes mellitus and hypertension. The mean logistic EuroSCORE was $18.7 \% \pm$ $15.3 \%$, which was equivalent to most registry data. The percentages of patients with NYHA Class II, III and IV were $18.6 \%, 52.5 \%$ and $28.8 \%$, respectively. The mean AVA was $0.70 \pm 0.19 \mathrm{~cm}^{2}$ and MPG was $45.0 \pm 16.3 \mathrm{mmHg}$ at baseline. The CT mean annulus area was $422.6 \pm 119.6 \mathrm{~mm}^{2}$ and mean annulus perimeter was $72.9 \pm 11.1 \mathrm{~mm}$ (Table I). In all, 40 (67.8\%) TAVI cases were done via TF access, 18 (30.5\%) via transapical access and 1 (1.7\%) via transaortic access. 30 (50.8\%) patients were implanted with 26-mm prosthetic valves, 21 (35.6\%) with 23-mm valves, 6 (10.2\%) with 29-mm valves, and the remaining two patients with $27-\mathrm{mm}$ and 31-mm valves each (Table I).

Survival rates after TAVI were $93.2 \%, 86.0 \%$ and $79.1 \%$ at 30 days, one year and two years follow-up, respectively. There were a total of nine deaths, all of which were cardiovascular-related; 
Table I. Baseline patient characteristics and procedural data $(n=59)$.

\begin{tabular}{|c|c|c|c|}
\hline Baseline & Mean \pm SD/No. (\%) & Baseline & Mean \pm SD/No. (\%) \\
\hline Characteristic & & Bicuspid aortic valve & $3(5.1)$ \\
\hline Age (yr) & $76.8 \pm 8.7$ & $\operatorname{LVEF}(\%)$ & $50.8 \pm 16.5$ \\
\hline Male gender & $36(61.0)$ & Aortic regurgitation* & \\
\hline STS score $(\%)$ & $6.9 \pm 5.8$ & None & $10(17.2)$ \\
\hline Logistic EuroSCORE (\%) & $18.7 \pm 15.3$ & Trivial & $7(12.1)$ \\
\hline Height $(\mathrm{cm})$ & $157.7 \pm 10.7$ & Mild & $26(44.8)$ \\
\hline Weight (kg) & $61.3 \pm 11.9$ & Moderate & $11(19.0)$ \\
\hline Body surface area $\left(\mathrm{m}^{2}\right)$ & $1.6 \pm 0.2$ & Severe & $4(6.9)$ \\
\hline Body mass index $\left(\mathrm{kg} / \mathrm{m}^{2}\right)$ & $24.9 \pm 4.3$ & MDCT data & \\
\hline NYHA functional class & & Maximum annulus diameter $(\mathrm{mm})$ & $25.6 \pm 2.5$ \\
\hline II & $11(18.6)$ & Minimum annulus diameter ( $\mathrm{mm}$ ) & $20.9 \pm 2.4$ \\
\hline III & $31(52.5)$ & Annulus area $\left(\mathrm{mm}^{2}\right)$ & $422.6 \pm 119.6$ \\
\hline IV & $17(28.8)$ & Annulus perimeter $(\mathrm{mm})$ & $72.9 \pm 11.1$ \\
\hline Coronary artery disease & $41(69.5)$ & Procedural data & \\
\hline Previous CABG & $8(13.6)$ & Implantation approach & \\
\hline Previous $\mathrm{PCl}$ & $21(35.6)$ & Implantation approach & \\
\hline Previous valve surgery & $3(5.1)$ & Transfemoral access & $40(67.8)$ \\
\hline Cerebral vascular disease & $3(5.1)$ & Transapical access & $18(30.5)$ \\
\hline Peripheral arterial disease & $16(27.1)$ & Transaortic access & $1(1.7)$ \\
\hline Chronic pulmonary disease & $15(25.4)$ & Procedure room & \\
\hline Diabetes mellitus & $28(47.5)$ & Cath lab & $8(13.6)$ \\
\hline Hypertension & $42(71.2)$ & Hybrid room & $51(86.4)$ \\
\hline Hyperlipidaemia & $40(67.8)$ & General anaesthesia & $59(100)$ \\
\hline eGFR & $53.6 \pm 25.0$ & Valve size $(\mathrm{mm})$ & \\
\hline Chronic kidney disease & $44(74.6)$ & 23 & $21(35.6)$ \\
\hline Pulmonary hypertension & $11(18.6)$ & 26 & $30(50.8)$ \\
\hline Echocardiographic data & & 27 & $1(1.7)$ \\
\hline Aortic valve area $\left(\mathrm{cm}^{2}\right)$ & $0.70 \pm 0.19$ & 29 & $6(10.2)$ \\
\hline Mean pressure gradient (mmHg) & $45.0 \pm 16.3$ & 31 & $1(1.7)$ \\
\hline
\end{tabular}

*Data calculated based on 58 patients. CABG: coronary artery bypass graft; eGFR: estimated glomerular filtration rate; EuroSCORE: European System for Cardiac Operative Risk Evaluation; LVEF: left ventricular ejection fraction; MDCT: multidetector computer tomography; NYHA: New York Heart Association; PCI: percutaneous coronary intervention; SD: standard deviation; STS: Society of Thoracic Surgeons

four of them were within the first 30 days. The most common cause of cardiovascular-related deaths was congestive cardiac failure. All-cause mortality rates for TF TAVI patients at 30 days, one year and two years were $2.5 \%, 7.5 \%$ and $14.8 \%$, respectively, versus $15.8 \%, 29.4 \%$ and $31.3 \%$, respectively, for non-TF TAVI patients (Table II).

At 30 days post TAVI, stroke occurred in $1.7 \%$ of patients, myocardial infarction in 1.7\%, life-threatening bleeding in 5.1\%, major bleeding in $3.4 \%$, AKI in $25.0 \%$ (of which $42.9 \%$ were Stage 1 , $14.3 \%$ Stage 2 and $42.9 \%$ Stage 3), major vascular complications in $5.1 \%$, and new permanent pacemaker implantations in $6.8 \%$ of patients. There were two cases of pericardial tamponade. $29.3 \%$ of TAVI patients were rehospitalised within one year, with about half being cardiovascular-related rehospitalisations. Composite outcomes were also measured according to VARC-2 definitions; we recorded rates of device success of $93.2 \%$, early safety (at 30 days) of $79.7 \%$, clinical efficacy (after 30 days) of $66.1 \%$ and time-related valve safety of $84.7 \%$ (Table II).

The Kaplan-Meier survival curves for the entire study population and that separated by the logistic EuroSCORE $(\leq 20 \%$ vs. $>20 \%$ ) are displayed in Figs. 1 a \& b, respectively. Patients with logistic EuroSCORE $>20 \%$ had significantly lower survival rates compared to those with logistic EuroSCORE $\leq 20 \%$ ( $p=0.0105)$. The most frequent TAVI complication was AKI within 30 days, and patients with AKI had significantly higher two-year all-cause mortality rates compared to those without (hazard ratio [HR] 5.3320, 90\% confidence interval [Cl] 1.6023-17.7439; $p=0.022$ ).

The Cox model identified logistic EuroSCORE and estimated glomerular filtration rate (eGFR) as the significant univariate predictors of two-year all-cause mortality (Table III). Other predictors, such as chronic kidney disease, age, male gender, severe PPM and non-TF approach, showed some evidence of increased two-year all-cause mortality risk, although they were not statistically significant. Multivariate Cox regression analysis, performed with published predictors, namely non-TF TAVI, age, gender, eGFR and severe PPM, identified non-TF TAVI as the only significant predictor of cardiovascular-related mortality at two years (HR 14.64, 90\% Cl 2.75-77.88; $\mathrm{p}=0.008$ ), after adjusting for all other predictors (Table III). There were no identifiable significant predictors for all-cause mortality at two years.

Prior to the TAVI procedure, $18.6 \%$ of the patients had NYHA Class II symptoms, while the remaining $81.3 \%$ had Class III 
Table II. Primary, secondary and composite outcomes after TAVI.

\begin{tabular}{|c|c|c|c|}
\hline \multirow[t]{2}{*}{ Outcome } & \multicolumn{3}{|c|}{ No. (\%) } \\
\hline & $\begin{array}{c}\text { TF } \\
(n=40)\end{array}$ & $\begin{array}{l}\text { Non-TF } \\
(n=19)\end{array}$ & $\begin{array}{c}\text { Total } \\
(n=59)\end{array}$ \\
\hline \multicolumn{4}{|c|}{$\begin{array}{l}\text { Primary (all-cause } \\
\text { mortality)* }\end{array}$} \\
\hline At 30 days & $1 / 40(2.5)$ & $3 / 19$ (15.8) & 4/59 (6.8) \\
\hline At $1 \mathrm{yr}$ & $3 / 40(7.5)$ & $5 / 17(29.4)$ & $8 / 57(14.0)$ \\
\hline At $2 \mathrm{yr}$ & $4 / 27(14.8)$ & $5 / 16(31.3)$ & $9 / 43(20.9)$ \\
\hline \multicolumn{4}{|c|}{ Secondary (30 days) } \\
\hline Stroke & & & $1 / 59(1.7)$ \\
\hline Myocardial ir & & & $1 / 59(1.7)$ \\
\hline All bleeding & & & $6 / 59(10.2)$ \\
\hline Life-threaten & ng & & $3 / 59(5.1)$ \\
\hline Major bleec & & & 2/59 (3.4) \\
\hline Minor bleec & & & $1 / 59$ (1.7) \\
\hline All acute kid & & & $14 / 56(25.0)$ \\
\hline Stage 1 & & & $6 / 14(42.9)$ \\
\hline Stage 2 & & & $2 / 14$ (14.3) \\
\hline Stage 3 & & & $6 / 14(42.9)$ \\
\hline Major vascul & cation & & $3 / 59(5.1)$ \\
\hline New perman & laker implant & tion & $4 / 59(6.8)$ \\
\hline New-onset a & ation & & $1 / 59(1.7)$ \\
\hline New-onset le & branch block & & $5 / 58(8.6)$ \\
\hline Other TAVI-re & iplications & & $3 / 59(5.1)$ \\
\hline Repeated pr & r valve-relatec & dysfunction & $1 / 58(1.7)$ \\
\hline Severe prost & ent mismatch & & $8 / 56$ (14.3) \\
\hline \multicolumn{4}{|c|}{ Paravalvular leak } \\
\hline None & & & $16 / 56(28.6)$ \\
\hline Trivial & & & $9 / 56$ (16.1) \\
\hline Mild & & & $29 / 56(51.8)$ \\
\hline Moderate & & & $2 / 56(3.6)$ \\
\hline Severe & & & 0 \\
\hline All-cause reh & tion (within 1 & & $17 / 58(29.3)$ \\
\hline Cardiovasc & & & $8 / 17(47.1)$ \\
\hline Non-cardio & lated & & $9 / 17$ (52.9) \\
\hline \multicolumn{4}{|l|}{ Composite } \\
\hline Device succe & & & $55 / 59(93.2)$ \\
\hline Early safety & & & $47 / 59(79.7)$ \\
\hline Clinical effic & 30 days) & & $39 / 59(66.1)$ \\
\hline Time-related & & & $50 / 59(84.7)$ \\
\hline
\end{tabular}

*Values are calculated based on available patient data. NYHA: New York Heart Association; TAVI: transcathether aortic valve implantation; TF: transfemoral

or IV symptoms. At the one-year follow-up, there was significant improvement in symptoms, with about $84.0 \%$ of patients returning to NYHA Class I and the rest in Class II (Fig. 2).

With regard to valve function, the risk of severe PPM was $14.3 \%$. At 30 days, the risks of moderate and severe PVL were $3.6 \%$ and $0 \%$, respectively. At the two-year follow-up, $22.7 \%$ of patients were found to have moderate PVL. The durability of the bioprosthetic valve performance confirms that AVA and MPG remained stable throughout the two-year follow-up (Fig. 3). AVA at baseline, 30 days and two years was $0.70 \pm 0.19 \mathrm{~cm}^{2}, 1.57 \pm$ $0.48 \mathrm{~cm}^{2}, 1.62 \pm 0.49 \mathrm{~cm}^{2}$, respectively. MPG at baseline, 30 days
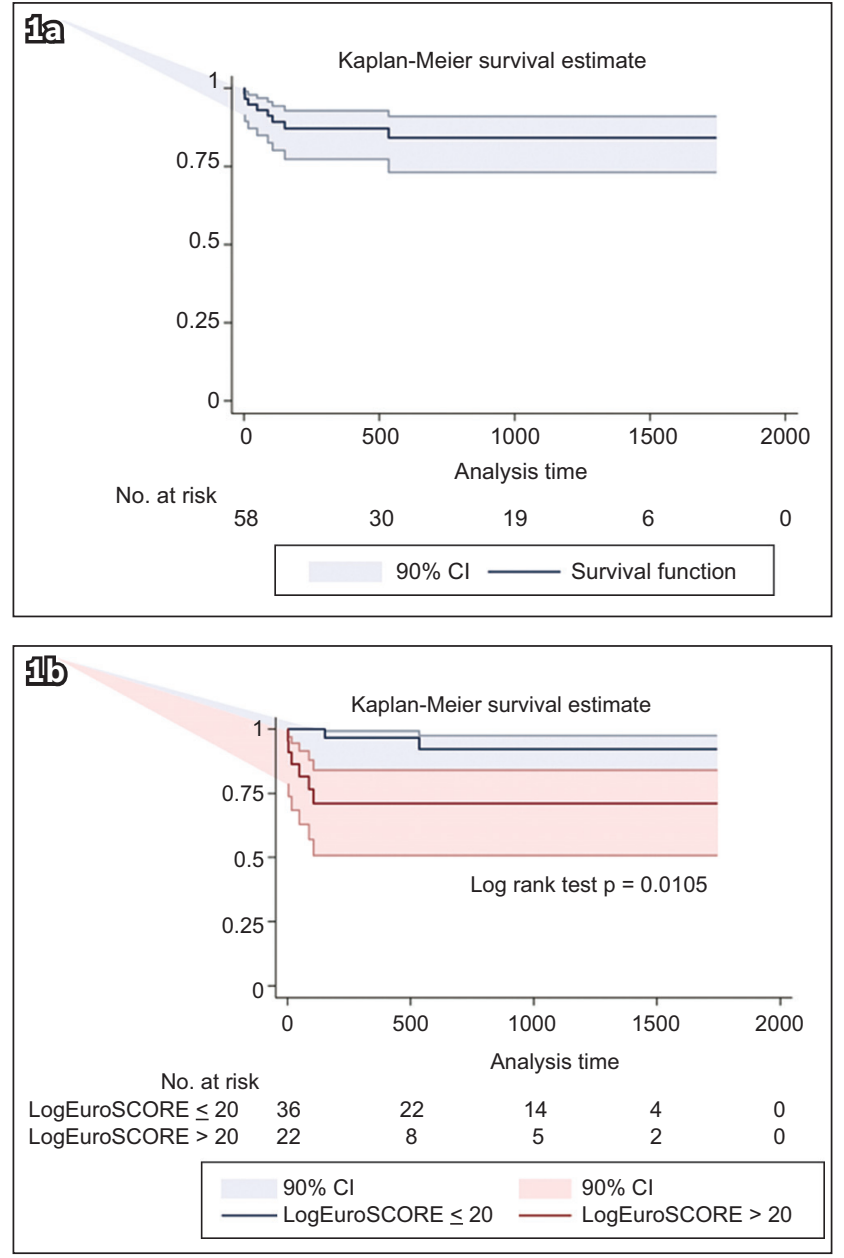

Fig. 1 Kaplan-Meier survival curves show (a) two-year survival and overall survival for all patients; and (b) two-year survival based on the logistic EuroSCORE and overall survival by logistic EuroSCORE $>20$ and $\leq 20$. $\mathrm{Cl}$ : confidence interval

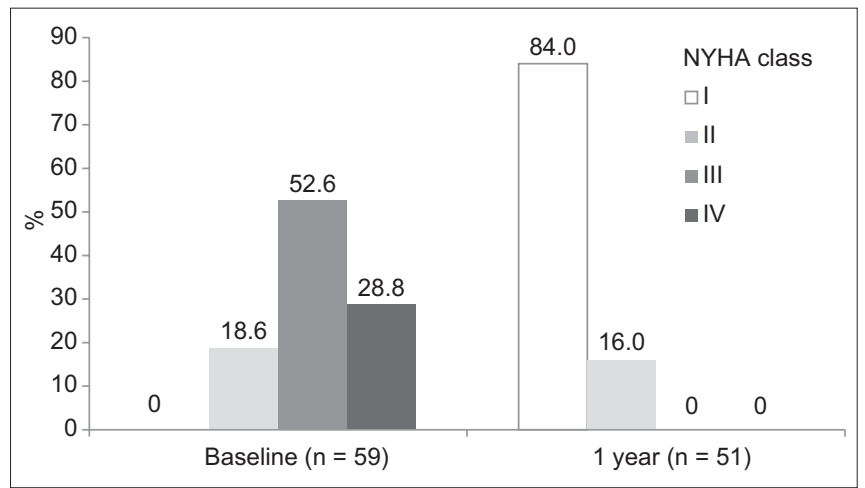

Fig. 2 Graph shows the New York Heart Association (NYHA) symptom classes throughout the one-year follow-up. All patients were observed at baseline and at one year, and classified according to NYHA Class I, II, III or IV.

and two years was $45.0 \pm 16.3 \mathrm{mmHg}, 10.9 \pm 4.5 \mathrm{mmHg}$ and $10.8 \pm 5.0 \mathrm{mmHg}$, respectively. Only one patient underwent surgical aortic valve replacement (SAVR) for endocarditis six months after the initial TAVI.

The TAVI experience was divided into two parts: first half $(\mathrm{FH}$, Patients 1-30) and second half (SH, Patients 31-59), according to the chronological procedural dates. The patient-selection process appeared to be constant throughout, with the mean logistic EuroSCORE showing no change between FH (18.7\%) 
Table III. Predictors of two-year mortality.

\begin{tabular}{|c|c|c|}
\hline Parameter & Hazard ratio $(90 \% \mathrm{Cl})$ & p-value \\
\hline \multicolumn{3}{|l|}{ Univariate predictor (all-cause) } \\
\hline Logistic EuroSCORE & $1.0667(1.0354-1.0989)$ & $<0.001$ \\
\hline eGFR & $0.9699(0.9454-0.9949)$ & 0.048 \\
\hline Chronic kidney disease & $1.7971(0.3019-10.6965)$ & 0.589 \\
\hline Age & $1.0528(0.9695-1.1432)$ & 0.305 \\
\hline Severe prosthesis-patient mismatch & $2.1080(0.5318-8.3559)$ & 0.373 \\
\hline Acute kidney injury & $5.3320(1.6023-17.7439)$ & 0.022 \\
\hline Delivery approach (non-transfemoral) & $2.7982(0.8722-8.9773)$ & 0.147 \\
\hline \multicolumn{3}{|c|}{ Multivariate predictor (cardiovascular-related) } \\
\hline Age & $1.30(1.03-1.63)$ & 0.063 \\
\hline Female gender & $0.70(0.11-46.27)$ & 0.888 \\
\hline Severe prosthesis-patient mismatch & $7.65(0.09-688.40)$ & 0.457 \\
\hline Non-transfemoral procedure & $14.64(2.75-77.88)$ & 0.008 \\
\hline \multicolumn{3}{|l|}{ Multivariate predictor (all-cause) } \\
\hline Age & $4.42(1.09-17.97)$ & 0.082 \\
\hline Female gender & $1.09(0.99-1.19)$ & 0.127 \\
\hline eGFR & $0.48(0.75-3.05)$ & 0.513 \\
\hline Severe prosthesis-patient mismatch & $0.98(0.95-1.01)$ & 0.269 \\
\hline Non-transfemoral procedure & $2.24(0.35-14.36)$ & 0.476 \\
\hline
\end{tabular}

$\mathrm{Cl}$ : confidence interval; eGFR: estimated glomerular filtration rate; EuroSCORE: European System for Cardiac Operative Risk Evaluation

and $\mathrm{SH}(18.6 \%)$. We also found no significant improvement in 30-day all-cause mortality rates between the two groups. When assessing the composite outcomes in $\mathrm{FH}$ with those in $\mathrm{SH}$, clinical efficacy improved from $60.0 \%$ to $72.4 \%(p=0.314)$, early safety improved from $73.3 \%$ to $86.2 \%(p=0.219)$, and time-related valve safety improved slightly from $83.3 \%$ to $86.2 \%$ ( $p=0.759)$; although some improvement was observed, the results were not statistically significant.

\section{DISCUSSION}

We postulated that the TAVI procedure presents certain unique challenges in an Asian population for a number of reasons. Firstly, iliofemoral vessel sizes are likely to be smaller in the Asian population due to the smaller Asian physique. Moreover, a lower BSA is an independent predictor of vessel size and tortuosity, which would have implications for TF TAVI patients (i.e. more vascular complications). ${ }^{(9)}$ Secondly, a Japanese study found that their patients had smaller annulus dimensions on multidetector CT as compared with their European counterparts, ${ }^{(10)}$ with implications for prosthesis-patient interactions. We also hypothesised that other factors, such as smaller left ventricular cavity size and increased frailty in Asian populations, may affect the clinical outcomes of TAVI. Currently, mid-term outcomes of TAVI on Asian patients have not been reported in any published clinical study. Therefore, we conducted this study to evaluate the mid-term safety and efficacy of the TAVI procedure using the newly revised VARC-2 criteria, comparing our results with those from well-established Western studies.
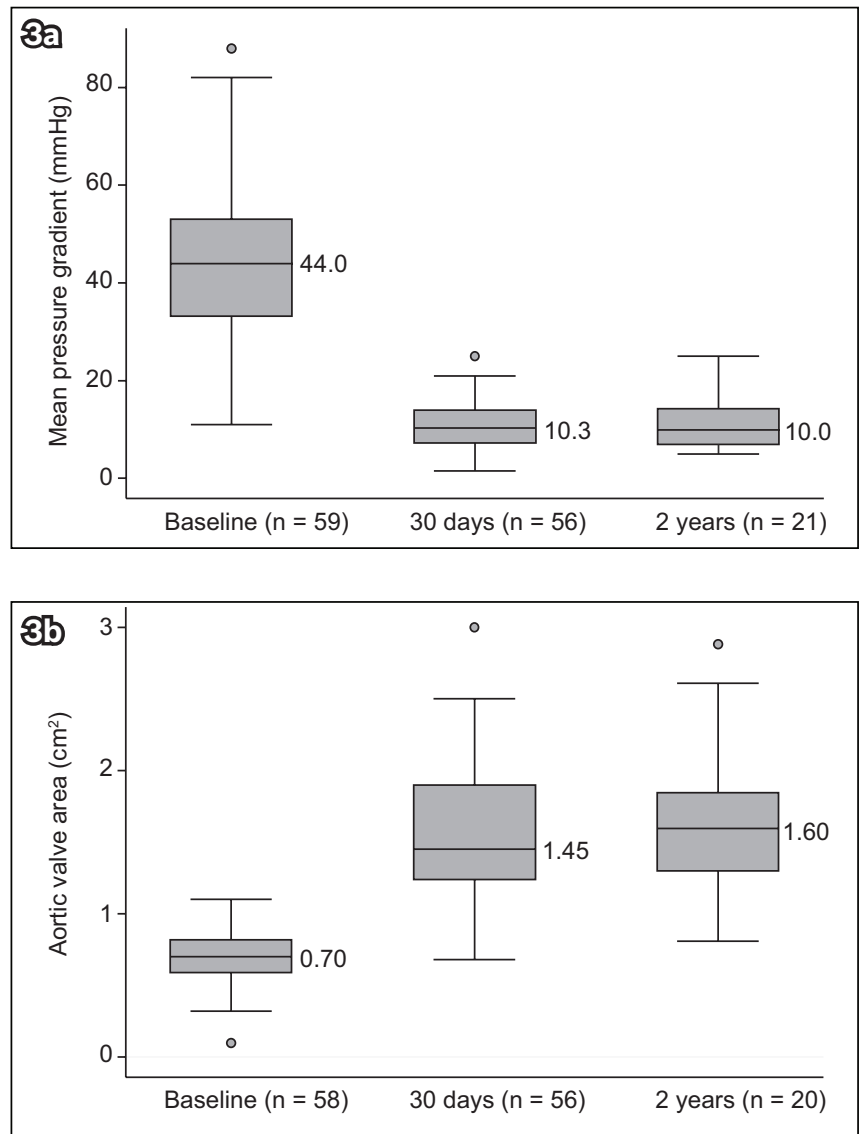

Fig. 3 Graphs show the echocardiographic data of all patients: (a) mean pressure gradient at baseline, 30 days and two years; and (b) aortic valve area at baseline, 30 days and two years. 
Table IV. Comparison of outcomes of transcatheter aortic valve implantation in the present study with those of the SOURCE XT registry and CoreValve trial. ${ }^{(13,19)}$

\begin{tabular}{|c|c|c|c|c|c|}
\hline \multirow[t]{2}{*}{ Study/No. of patients/Publication year } & \multicolumn{3}{|c|}{ All survival (\%) } & \multicolumn{2}{|c|}{ At 30 days $(\%)$} \\
\hline & 30-day & $1-y r$ & $2-y r$ & $\begin{array}{l}\text { Moderate/ } \\
\text { severe PVL }\end{array}$ & Major vascular complication \\
\hline Present study/59/2015 & 93.2 & 86.0 & 79.1 & 3.6 & 5.1 \\
\hline SOURCE XT/2,688/2015(13) & 93.7 & 80.6 & - & 5.3 & 6.5 \\
\hline \multicolumn{6}{|l|}{ RCT data } \\
\hline CoreValve/391/2015(19) & - & 85.8 & 77.8 & 9.0 & 5.9 \\
\hline
\end{tabular}

PVL: paravalvular leak; RCT: randomised controlled trial

We found that the mean BSA of our cohort was generally smaller than that in most European studies. The PARTNER trial reported that patients with a higher BSA had significantly higher rates of PPM. ${ }^{(11)}$ In line with that finding, the present study had relatively lower rates of severe PPM (14.3\%) as compared with the PARTNER trial (19.7\%), given the higher mean BSA in the latter. ${ }^{(11)}$ Moreover, patients in the PARTNER trial who did not have PPM had higher rates of post-procedural PVL. ${ }^{(1)}$ In contrast, our patients had lower rates of moderate or severe post-implant PVL at 30 days than patients in the PARTNER trial (3.6\% vs. 9.1\%, respectively). ${ }^{(12}$

This study reported a lower mean baseline logistic EuroSCORE $(18.7 \%)$ than most of the other published registries (mean logistic EuroSCORE > 20\%). The SOURCE XT registry, a European multicentre trial, reported a mean logistic EuroSCORE of 20.4\% $\pm 12.4 \%{ }^{(13)}$ It is important, however, to note that the logistic EuroSCORE was established as a risk assessment tool for open cardiac surgery. Therefore, some studies have indicated that it may not be very reliable when used alone to assess risk for TAVI patients; furthermore, its use has not been validated in Asian patients. ${ }^{(14-16)}$ Despite the lower mean logistic EuroSCORE, our study showed a higher burden of disease comorbidities compared to the SOURCE XT registry, ${ }^{(13)}$ especially in the incidence rates of coronary artery disease (69.5\% vs. $44.2 \%)$, peripheral vascular disease ( $27.1 \%$ vs. $21.2 \%$ ) and chronic kidney disease (74.6\% vs. $28.9 \%$ ). Thus, the results of this study must be interpreted with the context that our selected patients were at high risk.

In terms of primary outcome, the present study had comparable mortality rates as those reported by the SOURCE XT registry (Table IV). ${ }^{(13)}$ The following is a comparison of all-cause mortality rates between the present study and the SOURCE XT registry 30-day all-cause mortality rates: $6.8 \%$ vs. $6.3 \%$; 30-day all-cause mortality rates in TF TAVI group: $2.5 \%$ vs. $4.2 \%$; 30 -day all-cause mortality rates in non-TF TAVI group: $15.8 \%$ vs. $15.0 \%$; one-year all-cause mortality rates: $14.0 \%$ vs. $19.4 \%$; one-year all-cause mortality rates in TF TAVI group: $7.5 \%$ vs. $15.0 \%$; one-year allcause mortality rates in non-TF TAVI group: $29.4 \%$ vs. $27.1 \% .^{(13)}$ The present study's lower one-year all-cause mortality rates for the TF TAVI group emphasised the importance of patient selection because of the considerable mortality risk within the first year, despite a successful procedure. The present registry also had similar findings as other real-world, all-comers registries (30-day mortality rates: FRANCE II registry 9.7\%, German Aortic Valve Registry [GARY] transvascular TAVI 5.6\%, GARY transapical TAVI
9.0\%; one-year mortality rates: FRANCE II registry 24.0\%, GARY transvascular TAVI 20.7\%, GARY transapical TAVI 28.0\%). ${ }^{(17,18)}$ Interestingly, the two-year all-cause mortality rate in the present study was $20.9 \%$, which is one of the lowest observed for any TAVI trial in the real-world setting. This is comparable to a United States multicentre pivotal trial of 797 patients, which also used CoreValve self-expanding transcatheter bioprotheses, although it reported a slightly higher two-year all-cause mortality rate of $22.2 \%$. $^{(19)}$

Despite concerns related to the higher possibility of smallerbuilt Asian patients experiencing more frequent vascular or arrhythmic complications compared to patients of other ethnicities, we found that this was not the case for our patients. Ongoing studies have shown that smaller arterial diameters and a reduced difference in arterial and sheath diameters, together with extensive arterial wall calcification, can lead to a significantly increased risk of vascular complications. ${ }^{(20)}$ In the present study, the rate of major vascular complications within 30 days of TAVI was $5.1 \%$ as compared to the $6.5 \%$ reported in the SOURCE XT registry. ${ }^{(13)}$ Following TAVI, the rate of permanent pacemaker implantation in our patients (those without prior permanent pacemakers implantation) was lower than that reported in the PARTNER trial and the SOURCE XT registry $(6.8 \%, 8.8 \%$ and $9.5 \%$, respectively). ${ }^{(21)}$ These findings are in agreement with data on second-generation balloon expandable platforms. However, it is important to highlight that self-expanding valves generally have higher pacemaker complications than balloon expandable systems; moreover, the significant difference in mean ages reported for each study is likely to contribute to the difference in rate of conduction disease. Nevertheless, our findings are promising with regard to the future implications of TAVI in Asian patients, as complications such as cardiac conduction disturbances can lead to longer hospital stays and higher rates of mortality. ${ }^{(21)}$

The present study reported a cardiovascular-related rehospitalisation rate within one year of $13.8 \%$, which is deemed reasonable when compared to data from other studies. Moreover, we found significant improvement in patient symptoms, as determined by conventional NYHA classifications. Given these findings, it is reasonable to hypothesise that TAVI could be a potentially cost-effective therapy in Asians. This provides a strong impetus for conducting formal cost-effectiveness analyses in the near future, so as to guide health ministries to consider increasing funding for this therapy. 
Composite outcomes were measured according to VARC-2 definitions in this study. Généreux et al, ${ }^{(22)}$ who performed a meta-analysis of 3,519 patients from 16 studies, reported a $92.1 \%$ device success rate (according to previous VARC criteria), while the present trial found a device success rate of $93.2 \%$. The PREVAIL trial in Japan, which showed a device success rate of $91.9 \%$, concluded that this rate was adequate to deem TAVI an effective treatment for Japanese patients; ${ }^{(23)}$ however, it is noteworthy that the study defined device success less strictly than the VARC-2 criteria. We acknowledge that the VARC-2 criteria for assessment of device success can be challenging to attain in cohorts that underwent valve-in-valve procedures (although our study also involved non-valve-in-valve procedures), or cohorts with small body mass index. In addition, the meta-analysis reported an early safety rate (at 30 days) of $32.7 \%$, ${ }^{(23)}$ as compared to the $79.7 \%$ reported in the present study. Ikeda et al ${ }^{(24)}$ proposed that this composite endpoint, although important, may introduce 'background noise', as it consists of acute complications (such as stroke and $\mathrm{AKI}$ ) that may reflect the presence of multiple comorbidities in these high-risk patients despite a perfectly functioning valve. Furthermore, the present study found that clinical efficacy (after 30 days) was $66.1 \%$ and time-related valve safety was $84.7 \%$; these two composite outcomes have rarely been reported previously. We speculate that the former endpoint may vary greatly among studies, as one of its components involves the occurrence of hospital readmission, a variable that may be subject to bias due to different hospital systems and thresholds for rehospitalisation in each country. Overall, we considered our composite rates acceptable in comparison with those reported by our Asian and European counterparts, especially since they were obtained based on the more stringent VARC-2 definitions.

In terms of the haemodynamic performance of the valves, the SOURCE XT registry reported stable valvular function for a period of one year. ${ }^{(13)}$ Our study extended this finding and showed promising results over a two-year period, with significant and sustained improvements in MPG and AVA. Unfortunately, our sample size was too small to analyse the performance of each valve type and size used.

The multivariate prediction model in the present study showed that non-TF access was a significant predictor of cardiovascularrelated mortality at the two-year follow-up. Similarly, the PARTNER trial and SOURCE XT registry reported that the non-TF approach had one of the highest HRs for one-year mortality. The PARTNER trial proposed that the significant difference between TF and non-TF TAVI could be due to the higher logistic EuroSCORE and higher incidence of peripheral disease in their non-TF TAVI group. ${ }^{(25)}$ We found that patients who had a logistic EuroSCORE $>20 \%$ had a significantly higher mortality rate than those whose score was $\leq 20 \%$. Although the logistic EuroSCORE should not be used in isolation for TAVI risk assessment, it is plausible that a high logistic EuroSCORE of $>20 \%$ represents too high a risk for Asian patients undergoing TAVI. In order to improve patient screening and approach selection, we emphasise the need to develop a reliable TAVI-specific risk-assessment algorithm that encompasses the important baseline risk factors reported in the literature.
Previous reports have also suggested procedural experience as an independent predictor of procedural success and 30-day mortality. ${ }^{(26)}$ Although our study showed some improvement in early safety, clinical efficacy and time-related valve safety in the later cases (i.e. SH patients) of TAVI performed, the results were not statistically significant. This was probably due to the study's small sample size.

In high-risk patients, TAVI and SAVR lead to better outcomes as compared to medical management alone. The PARTNER trial showed that TAVI, in contrast to medical management, resulted in early and sustained improvements in transaortic MPG and AVA, which were associated with improvements in left ventricular systolic function and left ventricular mass reduction. Decisions regarding choice of treatment, however, should always be made in the context of a heart valve multidisciplinary team. ${ }^{(27)}$

Overall, this study has demonstrated that TAVI provides good mid-term outcome data and symptomatic improvement, with comparable procedural complication rates, for Asian patients. With the ongoing development of smaller TF-TAVI delivery sheaths, the risk of vascular complications may be further reduced. The increased use of three-dimensional CT measurement of aortic annulus, together with enhanced delivery systems that allow for repositioning of the valve for optimised valve placement, and the development of novel TAVI valves (such as SAPIEN 3) with special sealing cuffs and skirts, may also reduce the risk of PVL. ${ }^{(28)}$ In the SAPIEN 3 observational study, which recruited intermediate-risk patients, the propensity score analysis demonstrated that TAVI was superior to SAVR in the primary composite outcomes of mortality, strokes and moderate or severe aortic regurgitation. This is encouraging, as it suggests that TAVI may also be a suitable alternative for intermediate-risk patients. ${ }^{(29)}$

The main limitation of this study is its small sample size that provided low statistical power. Moreover, as this was not a randomised controlled trial, we were unable to conclude whether TF access or non-TF access was safer. Similarly, we could not determine if TAVI was preferred over SAVR, in terms of safety and efficacy, among our patients. There was also no independent clinical events committee to measure the outcomes of the study. In addition, no echocardiography core laboratory was used in the study; thus, all echocardiographic data was site-reported, which may increase the risk of measurement bias. Furthermore, it was challenging to assess the severity of PVL using transthoracic echocardiography. Lastly, although TAVI is a less invasive treatment for severe AS, no quality-of-life data was obtained. Nevertheless, we did use the conventional NYHA classification to monitor improvement in symptoms.

In conclusion, mid-term survival outcomes in Asian patients undergoing TAVI are reassuring and comparable with wellestablished European and North American studies. There are, however, unique clinical differences between Asian and Western populations. Multiple Western studies have also proposed that $\mathrm{TAVI}$, in comparison with SAVR, is more cost-effective in high-risk or inoperable patients, ${ }^{(30)}$ and future trials are needed to determine if this holds true for the Asian population. 


\section{REFERENCES}

1. Tay EL, Lew PS, Poh KK, et al. Demographics of severe valvular aortic stenosis in Singapore. Singapore Med J 2013; 54:36-9.

2. Kapadia SR, Leon MB, Makkar RR, et al; PARTNER trial investigators. 5-year outcomes of transcatheter aortic valve replacement compared with standard treatment for patients with inoperable aortic stenosis (PARTNER 1): a randomised controlled trial. Lancet 2015; 385:2485-91.

3. Kappetein AP, Head SJ, Généreux P, et al. Updated standardized endpoint definitions for transcatheter aortic valve implantation: the Valve Academic Research Consortium-2 consensus document. Eur Heart J 2012; 33:2403-18.

4. Baumgartner $\mathrm{H}$, Hung J, Bermejo J, et al; American Society of Echocardiography; European Association of Echocardiography. Echocardiographic assessmen of valve stenosis: EAE/ASE recommendations for clinical practice. J Am Soc Echocardiogr 2009; 22:1-23; quiz 101-2.

5. Murphy DT, Blanke P, Alaamri S, et al. Dynamism of the aortic annulus: Effect of diastolic versus systolic CT annular measurements on device selection in transcatheter aortic valve replacement (TAVR). J Cardiovasc Comput Tomogr 2016; 10:37-43.

6. Lichtenstein SV, Cheung A, Ye J, et al. Transapical transcatheter aortic valve implantation in humans: initial clinical experience. Circulation 2006; 114:591-6.

7. Webb JG, Chandavimol M, Thompson CR, et al. Percutaneous aortic valve implantation retrograde from the femoral artery. Circulation 2006; 113:842-50.

8. Bapat V, Khawaja MZ, Attia R, et al. Transaortic Transcatheter Aortic valve implantation using Edwards Sapien valve: a novel approach. Catheter Cardiovasc Interv 2012; 79:733-40.

9. Chiam PT, Koh AS, Ewe SH, et al. Iliofemoral anatomy among Asians: implications for transcatheter aortic valve implantation. Int J Cardiol 2013; 167:1373-9.

10. Watanabe Y, Hayashida K, Takayama M, et al. First direct comparison of clinical outcomes between European and Asian cohorts in transcatheter aortic valve implantation: the Massy study group vs. the PREVAIL JAPAN trial. J Cardiol 2014; 65:112-6.

11. Pibarot P, Weissman NJ, Stewart WJ, et al. Incidence and sequelae of prosthesispatient mismatch in transcatheter versus surgical valve replacement in high-risk patients with severe aortic stenosis: a PARTNER trial cohort--a analysis. J Am Coll Cardiol 2014; 64:1323-34.

12. Kodali S, Pibarot P, Douglas PS, et al. Paravalvular regurgitation after transcatheter aortic valve replacement with the Edwards sapien valve in the PARTNER trial: characterizing patients and impact on outcomes. Eur Heart J 2015; 36:449-56.

13. Schymik G, Lefèvre T, Bartorelli AL, et al. European experience with the secondgeneration Edwards SAPIEN XT transcatheter heart valve in patients with severe aortic stenosis: 1-year outcomes from the SOURCE XT Registry. JACC CardiovasC Interv 2015; 8:657-69.

14. Ben-Dor I, Gaglia MA Jr, Barbash IM, et al. Comparison between Society of Thoracic Surgeons score and logistic EuroSCORE for predicting mortality in patients referred for transcatheter aortic valve implantation. Cardiovasc Revasc Med 2011; 12:345-9.

15. D'Ascenzo F, Ballocca F, Moretti C, et al. Inaccuracy of available surgical risk scores to predict outcomes after transcatheter aortic valve replacement.
J Cardiovasc Med (Hagerstown) 2013; 14:894-8

16. Mack MJ. Risk scores for predicting outcomes in valvular heart disease: how useful? Curr Cardiol Rep 2011; 13:107-12.

17. Mohr FW, Holzhey D, Möllmann H, et al; GARY Executive Board. The German Aortic Valve Registry: 1-year results from 13,680 patients with aortic valve disease. Eur J Cardiothorac Surg 2014; 46:808-16.

18. Gilard $\mathrm{M}$, Eltchaninoff $\mathrm{H}$, lung $\mathrm{B}$, et al; FRANCE 2 Investigators. Registry of transcatheter aortic-valve implantation in high-risk patients. N Engl J Med 2012; 366:1705-15.

19. Reardon MJ, Adams DH, Kleiman NS, et al. 2-Year Outcomes in Patients Undergoing Surgical or Self-Expanding Transcatheter Aortic Valve Replacement. Im Coll Cardiol 2015; 66:113-21.

20. Czerwińska-Jelonkiewicz K, Michałowska I, Witkowski A, et al. Vascular complications after transcatheter aortic valve implantation (TAVI): risk and long-term results. J Thromb Thrombolysis 2014; 37:490-8.

21. Nazif TM, Dizon JM, Hahn RT, et al; PARTNER Publications Office. Predictors and clinical outcomes of permanent pacemaker implantation after transcatheter aortic valve replacement: the PARTNER (Placement of AoRtic TraNscathetER Valves) trial and registry. JACC Cardiovasc Interv 2015; 8(1 Pt A):60-9.

22. Généreux P, Head SI, Van Mieghem NM, et al. Clinical outcomes after transcatheter aortic valve replacement using valve academic research consortium definitions: a weighted meta-analysis of 3,519 patients from 16 studies. J Am Coll Cardiol 2012; 59:2317-26.

23. Sawa Y, Takayama M, Mitsudo K, et al. Clinical efficacy of transcatheter aortic valve replacement for severe aortic stenosis in high-risk patients: the PREVAIL JAPAN trial. Surg Today 2015; 45:34-43.

24. Ikeda K, Ho M, Kawahara M. Valve Academic Research Consortium Consensus report: the pharmaceutical and medical devices agency perspective. J Am Coll Cardiol 2011; 58:777

25. Lefèvre T, Kappetein AP, Wolner E, et al; PARTNER EU Investigator Group. One year follow-up of the multi-centre European PARTNER transcatheter heart valve study. Eur Heart J 2011; 32:148-57.

26. Gurvitch R, Tay EL, Wijesinghe N, et al. Transcatheter aortic valve implantation: lessons from the learning curve of the first 270 high-risk patients. Catheter Cardiovasc Interv 2011; 78:977-84.

27. Douglas PS, Hahn RT, Pibarot P, et al. Hemodynamic outcomes of transcatheter aortic valve replacement and medical management in severe, inoperable aortic stenosis: a longitudinal echocardiographic study of cohort B of the PARTNER trial. J Am Soc Echocardiogr 2015; 28:210-7.e1-9.

28. Hamm CW, Arsalan M, Mack MJ. The future of transcatheter aortic valve implantation. Eur Heart J 2016; 37:803-10.

29. Thourani VH, Kodali S, Makkar RR, et al. Transcatheter aortic valve replacement versus surgical valve replacement in intermediate-risk patients: a propensity score analysis. Lancet 2016 Apr 1. doi: 10.1016/S0140-6736. [Epub ahead of print].

30. Reynolds MR, Magnuson EA, Wang K, et al; PARTNER Investigators. Costeffectiveness of transcatheter aortic valve replacement compared with standard care among inoperable patients with severe aortic stenosis: results from the placement of aortic transcatheter valves (PARTNER) trial (Cohort B). Circulation 2012; 125:1102-9. 